Journal of

Molecular Microbiology

and Biotechnology

\title{
The Smallest Active Carbamoyl Phosphate Synthetase Was Identified in the Human Gut Archaeon Methanobrevibacter smithii
}

\author{
Elena Popa ${ }^{a} \quad$ Nirosha Perera $^{b} \quad$ Csaba Z. Kibédi-Szabóa ${ }^{a}$ Hedeel Guy-Evans ${ }^{b}$ \\ David R. Evans ${ }^{c}$ Cristina Purcarea ${ }^{a}$ \\ a Department of Microbiology, Institute of Biology Bucharest, Romanian Academy, Bucharest, Romania; \\ ${ }^{b}$ Department of Chemistry, Eastern Michigan University, Ypsilanti, Mich., and ${ }^{\mathrm{C}}$ Department of Biochemistry and \\ Molecular Biology, Wayne State University School of Medicine, Detroit, Mich., USA
}

\section{Key Words}

Carbamoyl phosphate synthetase $\cdot$ Ancestral form •

Methanobrevibacter smithii $\cdot$ Intestinal archaea • Evolution •

Obesity

\begin{abstract}
The genome of the major intestinal archaeon Methanobrevibacter smithii contains a complex gene system coding for carbamoyl phosphate synthetase (CPSase) composed of both full-length and reduced-size synthetase subunits. These ammonia-metabolizing enzymes could play a key role in controlling ammonia assimilation in M. smithii, affecting the metabolism of gut bacterial microbiota, with an impact on host obesity. In this study, we isolated and characterized the small (41 kDa) CPSase homolog from M. smithii. The gene was cloned and overexpressed in Escherichia coli, and the recombinant enzyme was purified in one step. Chemical crosslinking and size exclusion chromatography indicated a homodimeric/tetrameric structure, in accordance with a dimerbased CPSase activity and reaction mechanism. This small enzyme, MS-s, synthesized carbamoyl phosphate from ATP, bicarbonate, and ammonia and catalyzed the same ATP-dependent partial reactions observed for full-length CPSases. Steady-state kinetics revealed a high apparent affinity for ATP and ammonia. Sequence comparisons, molecular modeling, and kinetic studies suggest that this enzyme corre-
\end{abstract}

sponds to one of the two synthetase domains of the fulllength CPSase that catalyze the ATP-dependent phosphorylations involved in the three-step synthesis of carbamoyl phosphate. This protein represents the smallest naturally occurring active CPSase characterized thus far. The small $M$. smithii CPSase appears to be specialized for carbamoyl phosphate metabolism in methanogens.

Copyright $\odot 2012$ S. Karger AG, Basel

\section{Introduction}

In recent years, many studies [Dridi et al., 2011; Horz and Conrads, 2010; Nava et al., 2011; Plottel et al., 2011] have focused on the involvement of the human microbiome in human health and disease. While archaea colonize several different anaerobic niches within the human body [Belay et al., 1988, 1990], there is a single predominant species, Methanobrevibacter smithii, present in the human gut [Miller et al., 1982]. First isolated from feces, this methanogenic organism is a strict anaerobe that grows on a mixture of hydrogen and carbon dioxide [Balch et al., 1979].

Samuel and Gordon [2006] made the remarkable discovery that a synergic interaction in the colon between $M$. smithii and Bacteroides thetaiotaomicron, the major gut prokaryote, appears to have an important role in controlling host obesity [Samuel and Gordon, 2006]. Complex di-

\section{KARGER}

Fax +4161306 1234 E-Mail karger@karger.ch www.karger.com

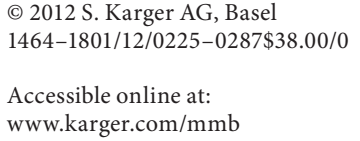

Dr. Cristina Purcarea

Department of Microbiology

Institute of Biology Bucharest, Romanian Academy

296 Splaiul Independentei, RO-060031 Bucharest (Romania)

E-Mail cristina.purcarea@ibiol.ro 
etary polysaccharides that cannot be metabolized by the human host are digested by enzymes in the gut microbiome [Stams, 1994]. Bacterial fermentation of polysaccharides yields short-chain fatty acids that provide as much as $10 \%$ of the daily caloric intake of the host. Archaeal methanogenesis facilitates bacterial fermentation by preventing the accumulation of $\mathrm{H}_{2}$ gas and other end products that would otherwise inhibit the process. Analysis of the cecal content of gnotobiotic mice colonized with M. smithii alone or together with $B$. thetaiotaomicron revealed that many genes that participate in the metabolism of these end products are upregulated when both organisms are present in the gut [Samuel et al., 2007]. Several lines of evidence suggest that obesity is associated with elevated levels of intestinal methanogenic archaea [Million et al., 2011]. Consequently, M. smithii is considered to be a potential therapeutic target in the treatment of obesity [Buck and Hansen, 2007]. The principle source of nitrogen in $M$. smithii is ammonia, resulting from the degradation of amino acids by the host or intestinal bacteria. M. smithii competes with $B$. thetaiotaomicron for the available ammonia, and when both organisms populate the gut, the transcription of several enzymes involved in ammonia transport and assimilation is upregulated in M. smithii. Although there have been numerous microbiological and physiological studies of human gut microbiota, little is known about the metabolism and key enzymes of the major archaeon in the gastrointestinal tract, M. smithii.

Carbamoyl phosphate synthetase (CPSase; EC 6.3.5.5) plays a major role in ammonia assimilation in all organisms. The enzyme catalyzes the synthesis of carbamoyl phosphate (CP), a common precursor of pyrimidine nucleotides, arginine, and, in terrestrial vertebrates, urea, where it represents the major means of eliminating ammonia. CPSases are multisubunit or multidomain proteins. Escherichia coli CPSase is composed of a $40-\mathrm{kDa}$ glutaminase (CarA, GLN) subunit which hydrolyzes glutamine and transfers ammonia to a $120-\mathrm{kDa}$ synthetase (CarB, SYN) subunit [Trotta et al., 1971]. The SYN subunit consists of two homologous domains having a nearly identical tertiary fold and active site residues that were thought to have evolved by ancestral duplication and fusion [Nyunoya and Lusty, 1983]. The amino half of the subunit, designated CPS.A, catalyzes the synthesis of carbamate from ATP, bicarbonate, and ammonia, while the carboxyl half, CPS.B, catalyzes CP synthesis from carbamate and a second ATP [Alonso and Rubio, 1995; Guy and Evans, 1996; Post et al., 1990]. E. coli CPSase GLN and SYN subunits associate to form heterodimers and tetramers [Anderson, 1986]. The partial reactions cata- lyzed by CPSase, first demonstrated for the E. coli enzyme [Anderson and Meister, 1965; Meister, 1989], are thought to occur universally in nearly all organisms:

CPS.A: (1) ATP + bicarbonate $\rightarrow$ carboxy phosphate + ADP; (2) carboxy phosphate $+\mathrm{NH}_{3} \rightarrow$ carbamate $+\mathrm{Pi}$

CPS.B: (3) ATP + carbamate $\rightarrow \mathrm{CP}+\mathrm{ADP}$

Each of the major CPSase domains is comprised of three subdomains designated A1, A2, A3 and B1, B2, B3 [Guillou et al., 1989; Guy and Evans, 1996; Post et al., 1990; Rubio et al., 1991]. The X-ray structure of E. coli CPSase [Thoden et al., 1997] showed that the A1-A2 and B1-B2 subdomains have nearly identical tertiary folds. A2 and B2 are catalytic subdomains, catalyzing reactions 1-2 and 3, respectively [Guy et al., 1997; Post et al., 1990; Thoden et al., 1997]. The A3 and B3 subdomains have distinctly different tertiary structures and functions. B3 is a regulatory subdomain that has been adapted to bind allosteric ligands [Rubio et al., 1991]. Remarkably, the active sites on the GLN, CPS.A and CPS.B domain are connected by a $96-\AA ̊$-long intramolecular tunnel that sequesters the labile intermediates within the $E$. coli enzyme complex [Thoden et al., 1997].

Although the structural organization of CPSases is very diverse, all are composed of homologous domains and subdomains and catalyze the same series of reactions. Generally, bacteria possess a single CPSase that catalyzes the formation of CP used for both arginine and pyrimidine nucleotide biosyntheses, while enteric and gram-positive bacteria contain two CPSases, each specific for one of these two metabolic pathways [Paulus and Switzer, 1979]. These enzymes are comprised of homologous SYN subunits of comparable size [Yang et al., 1997]. Most CPSases have a full-length SYN subunit consisting of fused CPS.A-CPS.B domains, but there are several organisms, including the thermophilic archaeon Methanocaldococcus jannaschii [Bult et al., 1996] and the hyperthermophilic eubacterium Aquifex aeolicus [Ahuja et al., 2001], that have a split carB gene encoding separate polypeptides corresponding to CPS.A and CPS.B domains. The only known exception is a $34-\mathrm{kDa}$ carbamate kinaselike CPSase of an entirely different sequence and tertiary structure found in the hyperthermophilic archaeon $P y$ rococcus furiosus [Durbecq et al., 1997; Legrain et al., 1995; Marina et al., 1998; Uriarte et al., 2001], and in Pyrococcus abyssi [Purcarea et al., 1996, 2001].

The genome of $M$. smithii contains carA and carB genes coding for a $40-\mathrm{kDa}$ GLN subunit and a $118-\mathrm{kDa}$ SYN subunit (MS-1, SYN1), respectively, that are similar to the CPSase subunits found in most bacteria [Popa et 
Fig. 1. MS-s sequence identity and domain structure. a Domain and subdomain organization of MS-s and the full-length SYN subunits. The full-length SYN domain consists of homologous CPS.A and CPS.B domains, each of which are comprised of subdomains designated A1, A2, A3 and B1, B2, B3, respectively, of the E. coli SYN subunit. b Protein sequence identity (\%) was calculated by pair-wise alignment of CPSase synthetase subunits, as indicated in Experimental Procedures. The proteins analyzed in both panels are: $M$. smithii small (MS-s) and large (MS-1) SYN; $M$. thermoautotrophicus small (MT-s) and large (MT-l) SYN, M. stadtmanae small (MST-s) and large (MST-l) SYN; E. coli (EC) SYN, and P. abyssi (PA) CK-like CPSase.

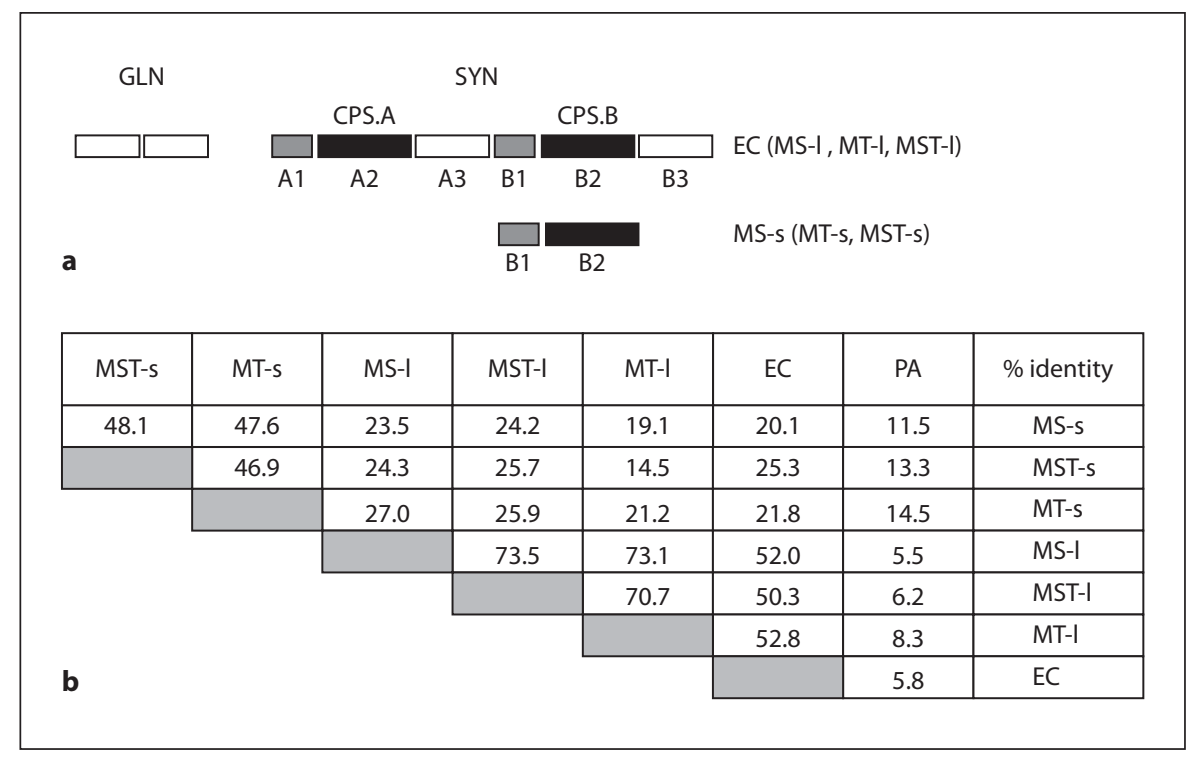

al., 2009]. Surprisingly, the genome also contains two genes coding for much smaller proteins that are homologous to the CPSase synthetase domain. One of these proteins (SYN2) consists of 391 residues $(45 \mathrm{kDa})$ with a sequence that resembles a truncated CPS.B domain, but it lacks active site residues that are known to participate in substrate binding and catalysis. Therefore, it is likely to be encoded by an inactive pseudogene. The second small CPSase homolog (MS-s, SYN3) is also homologous to a part of CPS.B and consists of 367 residues $(41 \mathrm{kDa})$ including the residues implicated in catalysis [Popa et al., 2009]. Prior to this report it was not known whether this protein has catalytic activity or is an inactive remnant of an unproductive gene duplication event.

We have isolated and characterized the small $M$. smithii CPSase MS-s, showing that it is catalytically active, synthesizing CP from $\mathrm{NH}_{3}, 2$ ATP, and bicarbonate by the same mechanism utilized by the large CPSases. This is the smallest functional CPSase discovered thus far and may more closely resemble the putative ancestral kinase that gave rise to this diverse family of enzymes.

\section{Results and Discussion}

\section{Sequence Analysis}

The large SYN subunit of the CPSase from the methanogens M. smithii (MS-1), Methanothermobacter thermoautotrophicus (MT-1), and Methanosphaera stadtmanae (MST-1) are 70-73\% identical to one another (fig. 1b). The alignment of the E. coli CPSase SYN subunit with the large CPSases MS-1, MT-1, and MST-1 gave sequence identities of 50-53\%, indicating that the CPSases from E. coli and methanogens are closely related to each other and to the SYN subunit of most prokaryotic and eukaryotic organisms.

The small CPSase from M. smithii, MS-s, is homologous to the CPS.B subdomain of E. coli but is significantly smaller, i.e. 367 versus 513 residues, respectively (fig. 1a), representing about a third of the full-length subunit (1,073 residues). Based on the deduced amino acid sequence, the protein has a calculated molecular mass of $41,403 \mathrm{Da}$ and an isoelectric point of 4.62. MS-s aligns with residues $560-947$ of the E. coli SYN subunit corresponding to the B1 and B2 subdomains of CPS.B [Goillou et al., 1989] (fig. 1a) with a score of $20.1 \%$ sequence identity (fig. 1b) and therefore lacks the B3 subdomain at the carboxyl end of the polypeptide.

MS-s is also homologous to the E. coli CPS.A domain, as expected, since the CPS.A and CPS.B domains of the E. coli enzyme are quite similar to one another (19.3\% identity, $34.5 \%$ similarity). However, the alignment score of MS-s is somewhat higher for CPS.B (20.1\% identity, $40.1 \%$ similarity) than for CPS.A, and the modeling program selected CPS.B as the best structural template. By analogy to the function of B3 in other CPSases, which have binding sites for both allosteric activators and inhibitors [Fresquet et al., 2000; Goillou et al., 1989; Guy and Evans, 1996; Rubio et al., 1991], the lack of this domain in MS-s suggests that this enzyme is unregulated. 
Fig. 2. Phylogenetic tree of CPS SYN subunits. The phylogenetic tree was constructed as indicated in Experimental Procedures, using the amino acid sequences of CPS SYN subunits or domains from the methanogens M. smithii small (MS-s) and large (MS-1), M. thermoautotrophicus small (MT-s) and large (MT-1), M. stadtmanae small (MST-s) and large (MST-l), M. jannaschii (MJ), A. aeolicus (AA), E. coli (EC), Helicobacter pylori (HP), the archaea Picrophilus torridus (PT), Sulfolobus acidocaldarius (SA), P. furiosus (PF), $P$. abyssi (PA) CK-like CPSase, Saccharomyces cerevisiae (SC) pyrimidine-specific enzyme, and the hamster pyrimidine biosynthetic multifunctional protein (CAD).

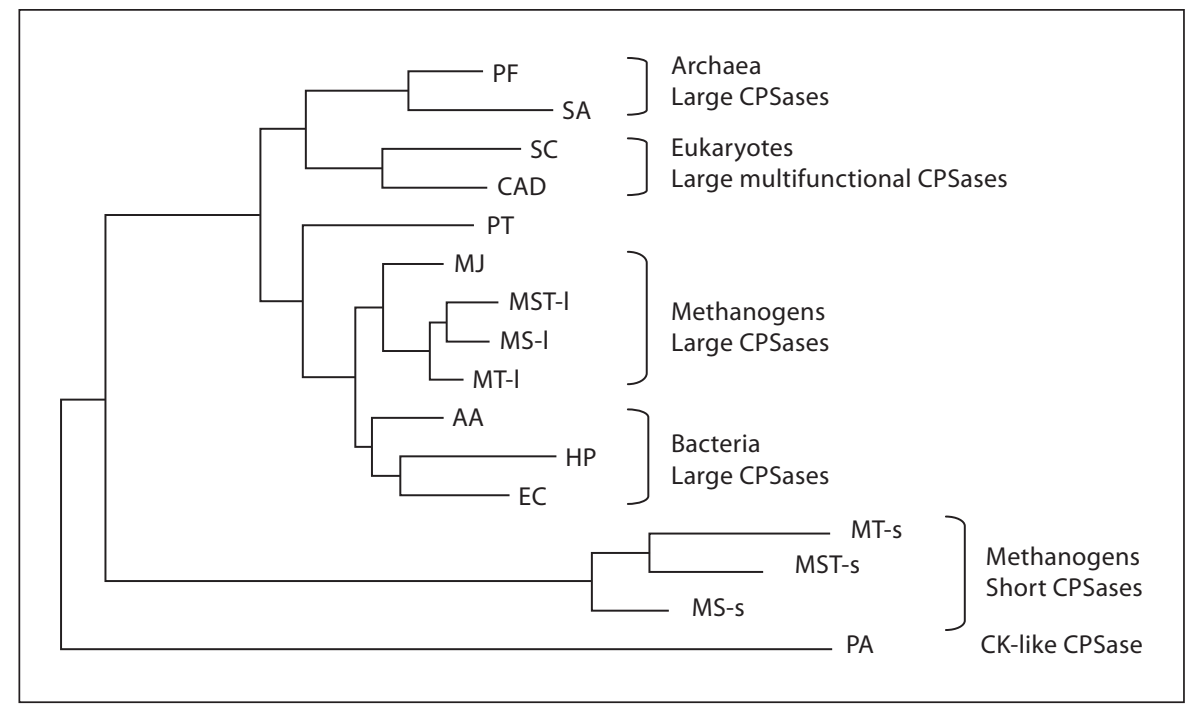

Open reading frames encoding large and small putative CPSases found in M. smithii are also present in the genomes of the thermophilic archaeon M. thermoautotrophicus and in the human intestinal archaeon M. stadtmanae; therefore, this unusual complement of CPSase genes may represent a common feature of methanogens. The overall sequences of the small CPSases from these three methanogens are well conserved (46.9-48.1\% identity) and are much less similar to the synthetase domain of E. coli CPSase (20.1-25.3\% identity).

Moreover, the deduced amino acid sequence of the small M. smithii CPSase has a limited overall identity score (19.1-24.2\%) with the corresponding SYN subunits of full-length CPSases from $E$. coli and methanogens, but an appreciably higher identity score (47.6-48.1\%) with the small CPSases from methanogens (fig. 1b). The large SYN subunit of the CPSase from the methanogens $M$. smithii (MS-1), M. thermoautotrophicus (MT-1), and M. stadtmanae (MST-1) are 70-73\% identical to one another (fig. 1b). The alignment of the E. coli CPSase SYN subunit with the large CPSases MS-1, MT-1, and MST-1 gave sequence identities of $50-53 \%$, indicating that the fulllength CPSases from E. coli and methanogens are closely related to each other and to the SYN subunit of most prokaryotic and eukaryotic organisms.

Since both MS-s and the CK-like CPSases are small $\mathrm{CP}$-synthesizing enzymes having a molecular mass of 41 $\mathrm{kDa}$ and $33 \mathrm{kDa}$, respectively, we investigated the possibility that MS-s may be more closely related to the CKlike CPSases than to the larger forms of the SYN CPSase subunits found in most bacteria. However, while the percent identity between MS-s and E. coli CPSase is relative- ly low (20.1\%), the percent identity between the MS-s and P. abyssi CK-CPSase is only $11.5 \%$. This difference is highly significant at these low levels of sequence similarity. Four randomized versions of the P. abyssi CK-CPSase sequence were generated of identical in size and amino acid composition but completely unrelated amino acid sequences. Sequence alignments yielded a percent identity between MS-s and the four randomized P. abyssi proteins of 7.4, 13.0, 14.1, and $15.1 \%$. This analysis suggests that MS-s and P. abyssi CPSase matches are coincidental and that the proteins are not homologs. This interpretation was reinforced by the conservation of $E$. coli CPSase active site residues and the kinetic studies described below.

The calculated phylogenetic tree (fig. 2) illustrates the relationship between the CPSase SYN subunit from methanogens, E. coli and other organisms belonging to all three domains of organisms, Bacteria, Archaea, and Eukarya. The small CPSases from the methanogens were the first to diverge, forming a separate branch. These proteins are more distantly related to the other bacterial, archaeal, and eukaryotic CPSases. Distinct branches cluster the eukaryotic CPSases from yeast (SC) and mammals (CAD), the archaeal large CPSases, and the bacterial fulllength CPSases. One unanticipated result is that CPSase from A. aeolicus (AA), an organism of ancient lineage which has a split gene encoding separate CPS-A and CPSB polypeptides [Ahuja et al., 2001], is a close relative of $E$. coli CPSase and other bacterial CPSases. Interestingly, the large methanogenic CPSases cluster together forming a separate lineage distinct from other bacterial and archaeal enzymes. The carbamate kinase-like CPSase from 


\begin{tabular}{|c|c|c|c|c|}
\hline CPS.A & $121 \mathrm{DF}$ & \multicolumn{3}{|c|}{ DAIDKAEDRRRFDVAMKKIGLETARSGIAHTMEEALAVAADVGFPCI IRPSFTMGGSGGGIAYN } \\
\hline MS-s & $97 \mathrm{R} z$ & \multicolumn{3}{|c|}{ RAVRLTSDKIKTKEFYNEIGVPTPQYQIL--AKDDFESKLKMEFPVVLKQGQGQGGKDIKVAES } \\
\hline CPS.B & $667 \mathrm{DF}$ & \multicolumn{3}{|c|}{$\underset{677}{\text { DAIDRAEDRERFQHAVERLKLKQPANATVTTIEMAVEKAKEIGYPLVVRPSYVLGGRAME IVYD }}$} \\
\hline CPS.A & $185 \mathrm{RH}$ & \multicolumn{3}{|c|}{ REEFEEICARGLDLSPTKELLIDES-L I I GWKEYEEMEVVRDKNDNCI IVCS IENFDAM-GI I HTGD } \\
\hline MS-s & $161 \mathrm{LI}$ & \multicolumn{3}{|c|}{ 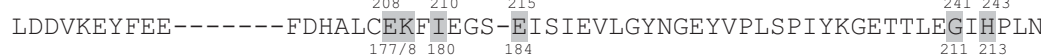 } \\
\hline CPS.B & $731 \mathrm{E} F$ & \multicolumn{3}{|c|}{ EADLRRYFQTAVSVSNDAPVLL-DHFLDDAVEVDVDAI-CDGEMVLIGGIMEH IEQA-GVHSGD } \\
\hline CPS.A & $248 \mathrm{SI}$ & \multirow{3}{*}{\multicolumn{3}{|c|}{ 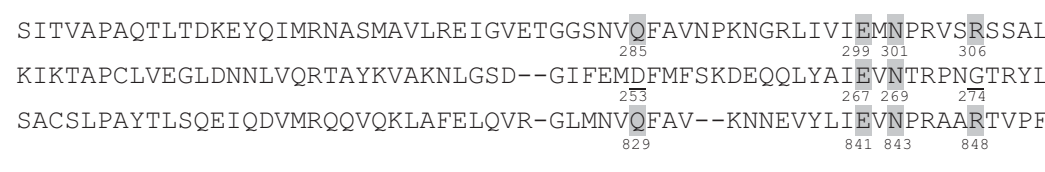 }} \\
\hline MS-s & $218 \mathrm{KJ}$ & & & \\
\hline CPS.B & $793 \mathrm{Sz}$ & & & \\
\hline & \multicolumn{2}{|c|}{ E. coli } & M. smithii & \multirow[t]{2}{*}{ Functional Interactions } \\
\hline & CPS.A & CPS.B & MS-s & \\
\hline & Arg129 & $($ Arg677) & Lys107 & $\alpha$ and $\beta$ phosphates AMP-PNP \\
\hline & Arg169 & Arg715 & Lys145 & $\alpha$ phosphate of AMP-PNP \\
\hline & Gly175 & Gly721 & Gly151 & $\gamma$ phosphate of AMP-PNP \\
\hline & Gly176 & Gly722 & Gly152 & $\beta$ phosphate of AMP-PNP \\
\hline & Glu208 & Asp753 & Glu177 & amino group of adenine ring \\
\hline & (Ser209) & His754 & Lys178 & amino group of adenine ring \\
\hline & Leu210 & Leu756 & Ile180 & purine ring \\
\hline & Glu215 & Glu761 & Glu184 & 2' and 3' hydroxyl of ribose \\
\hline & Gly241 & Gly786 & Gly211 & 2' hydroxyl of ribose \\
\hline & Gln285 & Gln829 & Asp253* & $\alpha$ phosphate of AMP-PNP/Mg ${ }^{2+}$ \\
\hline & Glu299 & Glu841 & Glu267 & binds $\mathrm{Mg}^{2+}$ \\
\hline & Asn301 & Asn843 & Asn269 & binds $\mathrm{Mg}^{2+}$ \\
\hline & Arg306 & Arg848 & Gly274* & $\gamma$ phosphate of AMP-PNP \\
\hline
\end{tabular}

Fig. 3. Active site conservation of MS-s. The alignment of MS-s with E. coli CPS.A and CPS.B domains was carried out as indicated in Experimental Procedures. The residues that interact with the bound substrates AMPPNP and $\mathrm{Mg}^{2+}$ and those that are part of the loop closing the active site identified in the X-ray structure of $E$. coli CPSase [Thoden et al., 1999] are indicated in both the

P. abyssi (PA) [Purcarea et al., 2001] is located on a distant branch of the phylogenetic tree, consistent with its distinctive sequence and structure.

The residues involved in substrate binding and catalysis in MS-s were identified based on X-ray structural analysis of $E$. coli CPSase with a nonhydrolyzable ATP analog [Thoden et al., 1999]. The alignment of M. smithii MS-s with the E. coli CPS.B and CPS.A domains (fig. 3) showed that all of the putative active site residues from one or the other domain have been conserved. The only exception is Arg848 in E. coli CPS.B that has been replaced in the archaeal enzyme by Gly 274 .

CPSase from M. smithii alignment and the table. The interacting residues in each E. coli subdomain are shaded grey and their position (residue number) is indicated. Residues in MS-s that are not conserved are underlined in the alignment and indicated by an asterisk in the table. The corresponding active site residues that are not interacting in CPS.A or CPS.B are enclosed in parentheses.

All of the active site residues identified in the X-ray structure of E. coli CPSase with a nonhydrolyzable ATP analog bond [Thoden et al., 1999] are located between residues 129-306 of the A2 subdomain and 677-848 of the B2 subdomain (fig. 3). Sequence alignment showed that of the 12 active site residues in CPS.A, which catalyze carbamate formation (reactions 1 and 2), all are conserved in the corresponding positions in CPS.B, which catalyzes CP synthesis (reaction 3). This high degree of conservation is consistent with the similarity of the reactions catalyzed by the two domains: ATP-dependent phosphorylations of bicarbonate and carbamate. The 
Fig. 4. Molecular modeling of MS-s. Superposition of the modeled three-dimensional structure of MS-s (red) with the E. coli CPSase 1BXR [Thoden et al., 1999] Xray structure (grey). The position of the ATP analog AMPPNP [Thoden et al., 1999] is shown (blue) bound to both CPS.A and CPS.B domains of the E. coli enzyme; the box indicates the region of the carbamate phosphorylation domain in E. coli CPSase, corresponding to B1-B2 subdomains.

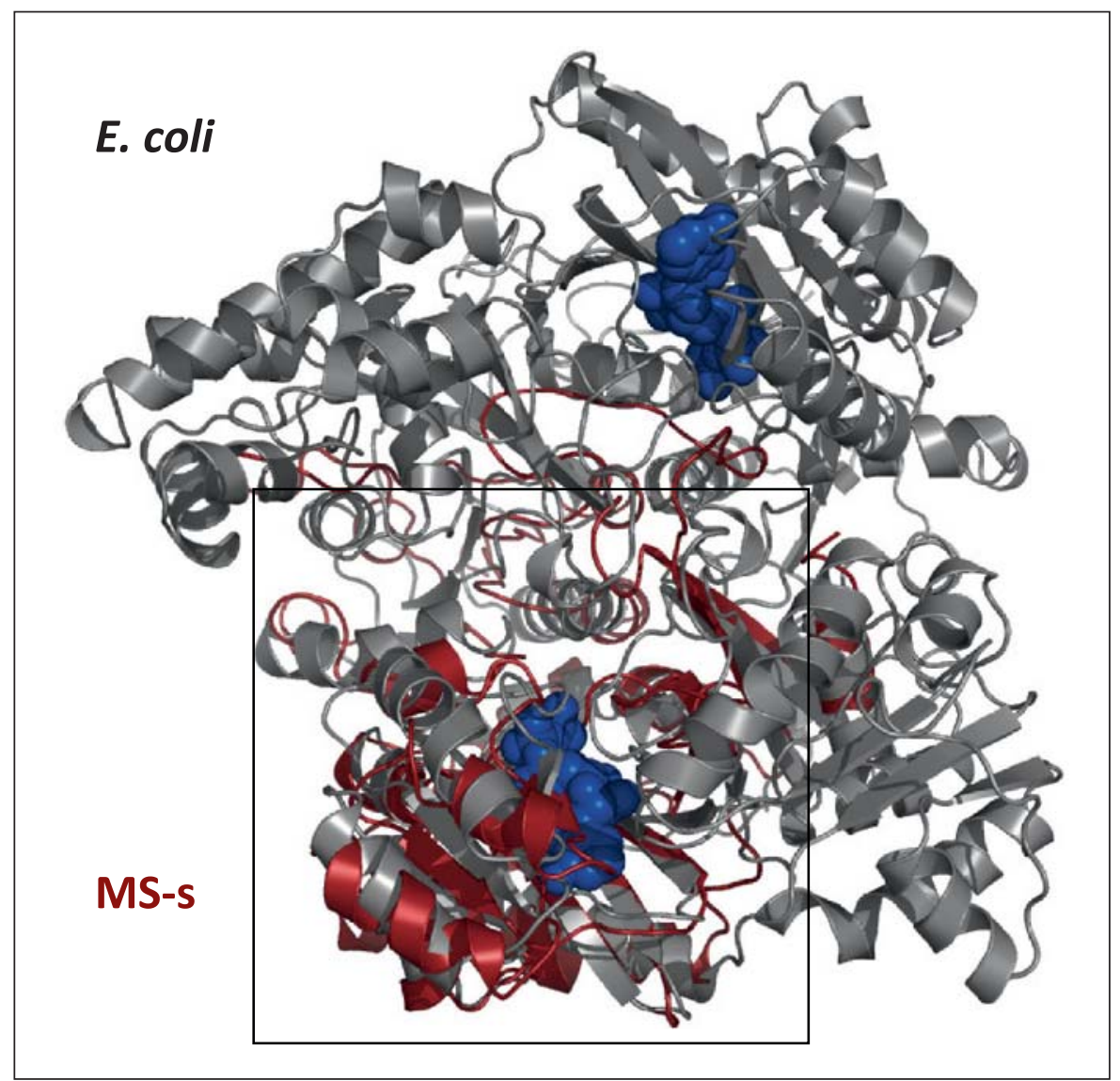

substrates are isosteric; the only difference is that one of the oxygens in bicarbonate is replaced by an $\mathrm{N}-\mathrm{H}$ in carbamate. When MS-s was included in the sequence alignment, 10 of these same 12 residues were found to be identical or highly conservative substitutions (K/R or L/I) with residues in CPS.A and/or CPS.B. Two MS-s residues, Asp253 and Gly274, are not conserved (fig. 3), but these are interactions with the backbone amide, not the side chain. As will be subsequently discussed, the alignments are consistent with the observation that MS-s can catalyze both carbamate and CP formation (reactions 1-3). It is significant that the putative active site residues are nearly perfectly conserved while the overall sequence identity to the E. coli subdomains is only $20 \%$.

\section{Molecular Modeling}

Modeling of the three-dimensional structure of MS-s was attempted using Modeller 9v9. The three-dimensional model was computed using the X-ray structure of the E. coli CPSase SYN subunit (pdb: 1BXR) as a template. The program fit (fig. 4) MS-s to the B1-B2 subdomains of the carbamate phosphorylation domain (CPS.B). The Ra- machandran plot of the model structure indicated that $95.6 \%$ of the residues are in most favored or allowed regions, $2.2 \%$ in generously allowed regions, and $2.2 \%$ in disallowed regions (data not shown). Due to the low sequence identity between MS-s and E. coli B1-B2 (20\%), the model statistics were poor [ERRAT2 score of 74 and $\mathrm{Z}$ score (ProSa) of -5.78$]$, precluding an accurate positioning of MS-s active site residues. However, the superposition of the model with $E$. coli CPSase gives a root mean square deviation of $1.66 \AA$ for $144 \alpha$-carbons and $1.66 \AA$ for backbone atoms, suggesting that the overall tertiary fold is likely to be approximately correct.

\section{Cloning, Expression, and Purification of \\ Recombinant MS-s}

M. smithii ATCC 35061 (DSM 861) cells were grown anaerobically at $37^{\circ} \mathrm{C}$ using an $\mathrm{H}_{2}: \mathrm{CO}_{2}$ gas mixture, as described in Experimental Procedures. Under these conditions, the culture reached the stationary phase after $200 \mathrm{~h}$ (data not shown). Chromosomal DNA was isolated in the presence of mutanolysin, as described in Experimental Procedures, yielding $86.5 \mathrm{mg} / \mathrm{ml}$ archaeal DNA. The MS-s 
carB gene (YP_001273061) coding for the M. smithii 367-residue CPSase was amplified by PCR, and the resulting DNA fragment was inserted into the pRSETC expression vector, using BamHI-NcoI restriction sites. This vector allows the expression of the recombinant CPSase with a $3-\mathrm{kDa}$ His-tag fused to the amino end of the protein. The resulting plasmid (pMS-s) was analyzed by double digestion with the cloning restriction enzymes BamHI-NcoI, and both strands were sequenced. The construct pMS-s encoding MS-s was expressed in E. coli BL21(DE3) by isopropyl- $\beta$-D-thiogalactopyranoside (IPTG) induction at $20^{\circ} \mathrm{C}$, and the recombinant MS-s was purified from the soluble fraction in a single step, by $\mathrm{Ni}^{2+}$ affinity chromatography (fig. 5a), as indicated in Experimental Procedures. The yield of the purified recombinant MS-s obtained was 14 $\mathrm{mg} / \mathrm{l}$ of culture. SDS-PAGE analysis of the purified protein gave the expected molecular mass of $44 \mathrm{kDa}$, taking into consideration the fused 3-kDa His-tag polypeptide.

\section{Oligomeric Structure}

The oligomeric structure of MS-s was assessed by chemical cross-linking with dimethyl suberimidate [Davies et al., 1970]. The time course of the cross-linking reaction (fig. 5b) shows that monomeric MS-s gradually disappeared as the reaction proceeded, and was converted into two discrete high molecular species, possibly a tetramer and a higher oligomeric species. The formation of sharp bands in the gels argues against nonspecific association of the monomers which would tend to give a broad, highly heterogeneous species forming a smear.

E. coli CPSase monomers associate to form dimers and tetramers [Kim and Raushel, 2001]. Since MS-s is homologous to the truncated CPS.B domain, the dimer would correspond to a full-length CPS.A-CPS.B synthetase monomer without the A3 and B3 subdomains. The MS-s tetramer would be equivalent to a dimer of the $E$. coli synthetase domain again without A3 and B3. The expected molecular mass of the MS-s dimer and tetramer would be 88 and $176 \mathrm{kDa}$, respectively, taking into account the appended 3-kDa His-tag polypeptide. Failure to observe the dimeric species is not unusual during chemical crosslinking and can occur if the residues involved in forming the cross-links are not optimally positioned so that the rate of cross-linking of the tetramer and higher oligomers occurs much more rapidly than the dimer.

The oligomeric structure of MS-s was further investigated by size exclusion chromatography on a calibrated Hiload 16/60 superdex 200 column (fig. 5c). Two protein peaks were observed by measuring the absorbance at 280 $\mathrm{nm}$ and SDS-PAGE analysis. Calibration of the column with known proteins gave a molecular mass of $91 \mathrm{kDa}$ and $185 \mathrm{kDa}$ for these two peaks, in good agreement with the expected size of the MS-s dimer $(88 \mathrm{kDa})$ and tetramer $(176 \mathrm{kDa})$. No monomeric MS-s was present, indicating that the protein associates to form homodimers and dimers of dimers.

\section{Catalytic Activity of MS-s}

The ammonia-dependent CPSase activity of the recombinant M. smithii CPSase MS-s was measured in the coupled reaction with ATCase, as indicated in Experimental Procedures. The enzyme catalyzes CP synthesis from ATP, ammonium chloride, and bicarbonate, with a specific activity at $37^{\circ} \mathrm{C}$ of $77 \pm 5.9 \mathrm{nmol} / \mathrm{min} / \mathrm{mg}$. Temperature had a very limited effect on the activity of MS-s, as the specific activity only decreased to $58.3 \pm 4.2 \mathrm{nmol} /$ $\mathrm{min} / \mathrm{mg}$ when the assays were conducted at $22^{\circ} \mathrm{C}$.

This small M. smithii CPSase also catalyzed the ATPdependent partial reactions in accordance with the threestep mechanism demonstrated for E. coli CPSase [Kothe et al., 2005; Meister, 1989] (table 1). The bicarbonate-dependent ATPase reaction in the presence (reactions 1 and 2) and absence (reaction 1 only) of ammonia measured at $22^{\circ} \mathrm{C}$ showed a ratio of $1.7: 1$, indicating that 2 ATP molecules are consumed in the $\mathrm{CP}$ synthesis when all of the substrates are present. Moreover, the CP-dependent ATP synthetase reverse reaction rate is very low $(4.52 \mathrm{nmol} /$ $\mathrm{min} / \mathrm{mg}$ ) relative to the ATP hydrolysis, representing $8 \%$ of the forward ATPase reaction in the presence of ammonia, as in the case of A. aeolicus CPSase [Ahuja et al., 2001].

Enterococcus faecium carbamate kinase [Marina et al., 1998] does not catalyze the first partial reaction, i.e. the bicarbonate-dependent ATP hydrolysis in the absence of ammonia, whereas the second partial reaction measured in the reverse direction as a CP-dependent ATP synthesis occurs at a very high rate (table 1). In contrast, for MS-s, like E. coli CPSase [Rubio et al., 1987], the rate of the bicarbonate-dependent ATP hydrolysis is high relative to the CP-dependent ATP synthesis activity. The kinetic studies provided additional strong evidence that MS-s is a canonical CPSase, not a carbamate kinase.

\section{Steady-State Kinetic Parameters}

Substrate saturation curves for $\mathrm{ATP}, \mathrm{NH}_{4} \mathrm{Cl}$, and $\mathrm{NaHCO}_{3}$ of the recombinant MS-s were measured at $37^{\circ} \mathrm{C}$ (data not shown), and the kinetic parameters were calculated from the least squares fit to either the Michaelis-Menten or the Hill equation. While both $\mathrm{NH}_{4} \mathrm{Cl}$ and $\mathrm{NaHCO}_{3}$ saturation curves follow Michaelis-Menten kinetics, the ATP saturation curve is slightly sigmoidal, with 

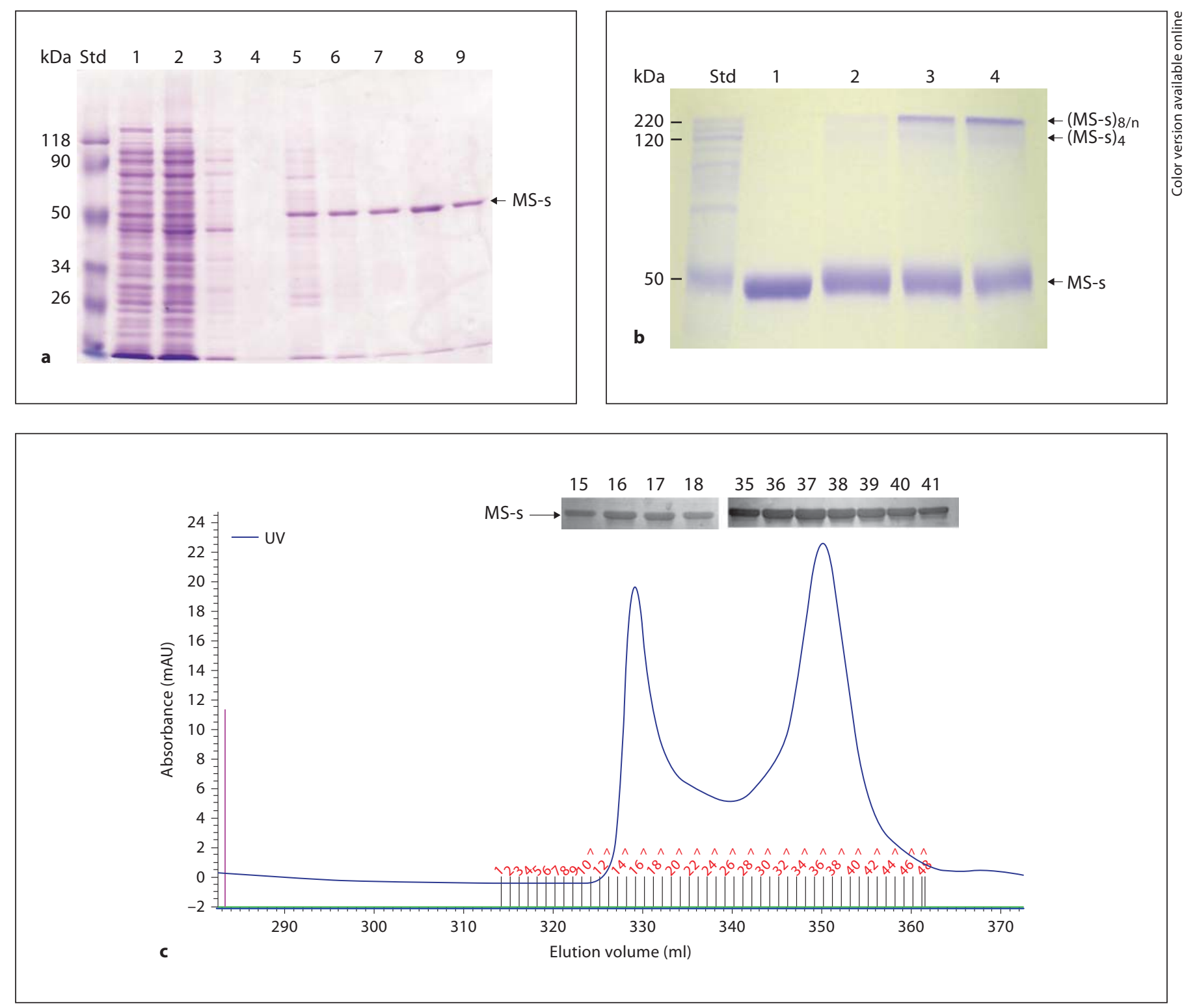

Fig. 5. MS-s purification and oligomeric structure determination. a Purification of the recombinant MS-s was carried out by affinity chromatography on $\mathrm{Ni}^{2+}$-Probond resin as described in Experimental Procedures, and the elution fractions were analyzed by $12 \%$ SDS-PAGE. The lanes correspond to the molecular weight marker (Std), fractions 1-3 eluted with TN buffer $(50 \mathrm{mM}$ TrisHCl, pH 8, $200 \mathrm{mM} \mathrm{NaCl}$ ), fractions 4-6 eluted with TN buffer containing $50 \mathrm{~mm}$ imidazole, and fractions 7-9 eluted with TN buffer containing $100 \mathrm{~mm}$ imidazole. b Chemical cross-linking was carried out at $22^{\circ} \mathrm{C}$ for $0,5,10$, and 15 min (lanes 1-4) using $20 \mu \mathrm{g}$ recombinant MS-s in $100 \mathrm{mM}$ TEA buffer, $\mathrm{pH}$ 8.5, $100 \mathrm{mM}$ $\mathrm{NaCl}$, and $10 \mathrm{~mm}$ dimethylsuberimidate. The reaction mixture was quenched with $100 \mathrm{~mm}$ TrisHCl, $\mathrm{pH} 8$, and analyzed by $10 \%$ SDS-PAGE. c Size exclusion chromatography was carried out as indicated in Experimental Procedures. The elution profile was obtained by measuring the absorbance at $280 \mathrm{~nm}$. The peak fractions (fractions 15-18 and 35-41) were analyzed by $10 \%$ SDS-PAGE and found to contain MS-s. a Hill coefficient of $n_{H}=1.4 \pm 0.1$. The steady-state kinetic parameters (table 2 ) indicated a relatively high apparent affinity for ATP $\left(K_{m}=0.61 \mathrm{mM}\right), 2$ - and 12-fold higher than the $\mathrm{K}_{\mathrm{m}}(\mathrm{ATP})$ of the E. coli SYN subunit $(1.3 \mathrm{mM})[\mathrm{Ru}-$ bio et al., 1987] and A. aeolicus CPS.A-CPS.B complex
(7.43 mM) [Ahuja et al., 2001], respectively. The $\mathrm{k}_{\text {cat }}$ value for this substrate $\left(0.043 \mathrm{~s}^{-1}\right)$ is 170 -fold lower than the corresponding value $\left(7.3 \mathrm{~s}^{-1}\right)$ for the $E$. coli SYN subunit $[\mathrm{Ru}-$ bio et al., 1987]. The apparent second-order rate constant $\mathrm{k}_{\text {cat }} / \mathrm{K}_{\mathrm{m}}$ for ATP $\left(70.5 \mathrm{M}^{-1} \mathrm{~s}^{-1}\right)$ is 21-23-fold higher than that 
for the other two substrates $\mathrm{NH}_{4} \mathrm{Cl}$ and $\mathrm{NaHCO}_{3}$, indicating a high efficiency for ATP reaction. Moreover, this second-order rate constant is 79 -fold lower than that of the E. coli SYN subunit $\left(5,600 \mathrm{M}^{-1} \mathrm{~s}^{-1}\right)$. Surprisingly, the $\mathrm{K}_{\mathrm{m}}\left(\mathrm{NH}_{4} \mathrm{Cl}\right)$ value of MS-s $(15.6 \mathrm{mM})$ is 13-fold lower than that for the E. coli enzyme (211 mM) [Huang and Raushel, 2000], indicating a high affinity for ammonia of this enzyme, while the value of $\mathrm{K}_{\mathrm{m}}\left(\mathrm{NaHCO}_{3}\right)$ is comparable to that of the E. coli SYN subunit (10.8 mM). Furthermore, this small M. smithii CPSase shows similar $\mathrm{k}_{\mathrm{cat}}$ and $\mathrm{k}_{\mathrm{cat}} /$ $\mathrm{K}_{\mathrm{m}}$ values for both $\mathrm{NaHCO}_{3}$ and $\mathrm{NH}_{4} \mathrm{Cl}$ substrates. While MS-s clearly catalyzed CP synthesis, the $\mathrm{k}_{\mathrm{cat}}$ values were surprisingly low compared to other CPSases. Whether the kinetic parameters accurately reflect the intrinsic activity or are the result of instability of the protein or other factors involved in the assay is under investigation.

\section{Mechanism of CP Synthesis}

Besides the canonical CPSases, the only other enzymes that have been found to synthesize CP are the archaeal carbamate kinases from the hyperthermophilic organisms, $P$. furiosus and $P$. abyssi. These enzymes use ATP and carbamate formed chemically from ammonia and bicarbonate to synthesize CP. Several lines of evidence suggest that MS-s is much more closely related to the longer CPSases found in all mesophillic organisms: (1) MS-s is significantly larger $(41 \mathrm{kDa})$ than either carbamate kinase or the hyperthermophilic enzymes ( $33 \mathrm{kDa})$; (2) MS-s exhibits no statistically significant sequence similarity to carbamate kinases; (3) the residues implicated in catalysis in E. coli CPSase are highly conserved in MS-s, whereas the enzyme lacks the carbamate kinase active site residues; (4) carbamate kinases do not catalyze the first partial reaction, the bicarbonate-dependent ATPase activity, but rapidly catalyze the CP-dependent ATP synthesis, while the reverse is observed for MS-s; (5) the stoichiometry of the reaction catalyzed by carbamate kinases is 1 mol of ATP consumed in CP synthesis, whereas 2 ATPs are required for the $\mathrm{CP}$ synthesis catalyzed by MS-s, and (6) the levels of ammonia are likely to be too low in the nitrogen-limited environment of the gut to provide sufficient carbamate to sustain significant CP synthesis. Determination of the three-dimensional structure of MS-s, now underway, should provide definitive evidence that the protein is more closely related to the mesophillic CPSases than carbamate kinase.

The question then arises of how a protein one third the size of the synthetase domain of $E$. coli CPSase can catalyze the complex series of reactions (reactions 1-3) involved in CP synthesis. A model is proposed (fig. 6) that
Table 1. ATP-dependent partial reactions

\begin{tabular}{|c|c|c|c|}
\hline \multirow[t]{2}{*}{ Enzyme } & \multicolumn{2}{|c|}{$\begin{array}{l}\mathrm{HCO}_{3}^{-} \text {-dependent } \\
\text { ATPase, } \mathrm{nmol} / \mathrm{min} / \mathrm{mg}\end{array}$} & \multirow{2}{*}{$\begin{array}{l}\text { CP-dependent } \\
\text { ATP synthetase } \\
\mathrm{nmol} / \mathrm{min} / \mathrm{mg}\end{array}$} \\
\hline & $+\mathrm{NH} 3$ & $-\mathrm{NH}_{3}$ & \\
\hline M. smithii MS-s & $58 \pm 4$ & $35 \pm 2$ & $4 \pm 1$ \\
\hline E. faecium CKase ${ }^{*}$ & 427 & 8 & 4,533 \\
\hline E. coli CPSase $\mathrm{C}^{* *}$ & $2,000 \pm 400$ & $400 \pm 50$ & $390 \pm 50$ \\
\hline
\end{tabular}

ADP and ATP formation catalyzed by M. smithii small CPSase (MS-s) was measured at $22^{\circ} \mathrm{C}$ as indicated in the Experimental Procedures. The specific activity and standard deviations were calculated from three experiments. ${ }^{*}$ Results from [Marina et al, 1998]. ${ }^{* *}$ Results from [Miran et al., 1991]

Table 2. Steady state kinetic parameters of MS-s

\begin{tabular}{lllll}
\hline Substrate & $\begin{array}{l}\mathrm{K}_{\mathrm{m}} \\
\mathrm{mM}\end{array}$ & $\begin{array}{l}\mathrm{V}_{\max }, \mathrm{nmol} / \\
\mathrm{min} / \mathrm{mg}\end{array}$ & $\begin{array}{l}\mathrm{k}_{\mathrm{cat}} \\
\mathrm{s}^{-1}\end{array}$ & $\begin{array}{l}\mathrm{k}_{\mathrm{cat}} / \mathrm{K}_{\mathrm{m}} \\
\mathrm{M}^{-1} \cdot \mathrm{s}^{-1}\end{array}$ \\
\hline $\mathrm{ATP}^{*}$ & $0.61 \pm 0.01$ & $62 \pm 1$ & $0.043 \pm 0.003$ & 70.5 \\
$\mathrm{NH}_{4} \mathrm{Cl}$ & $15.6 \pm 2.6$ & $75 \pm 3$ & $0.052 \pm 0.003$ & 3.3 \\
Bicarbonate & $14.5 \pm 3.6$ & $64 \pm 7$ & $0.044 \pm 0.006$ & 3.0 \\
\hline
\end{tabular}

The steady state kinetic parameters were determined at $37^{\circ} \mathrm{C}$, from the ATP, ammonium chloride and sodium bicarbonate saturation curves of MS-s $(10 \mathrm{mg})$ in the coupled reaction with A. aeolicus ATCase as indicated in the Experimental Procedures.

* The sigmoidal ATP saturation curve was fit to the Hill equation, so the $K_{m}$ was replaced with $[S] 0.5$. The steady state kinetic parameters and standard deviations were obtained by a least squares fit of eight data points in each saturation curve.

takes into consideration the overall structural and functional characteristics of MS-s, the smallest naturally occurring active CPSase identified so far. As discussed above, MS-s can catalyze both partial reactions, the formation of carbamate from ATP, ammonia, and bicarbonate and the ATP-dependent synthesis of CP from carbamate. In principle, the carbamate synthesized once released from the complex could react with a second ATP to form CP (fig. 6a), but this process would be expected to be inefficient because carbamate is hydrolyzed in the aqueous milieu to ammonia and bicarbonate, setting up in effect a futile cycle resulting in wasteful ATP hydrolysis. We propose instead that the functional unit is the MS-s dimer (fig. 6b). In this species, the two MS-s monomers would be related to each other by a two-fold axis of symmetry exactly analogous to the fused CPS.A and CPS.B domain in the E. coli enzyme. The model also pos- 


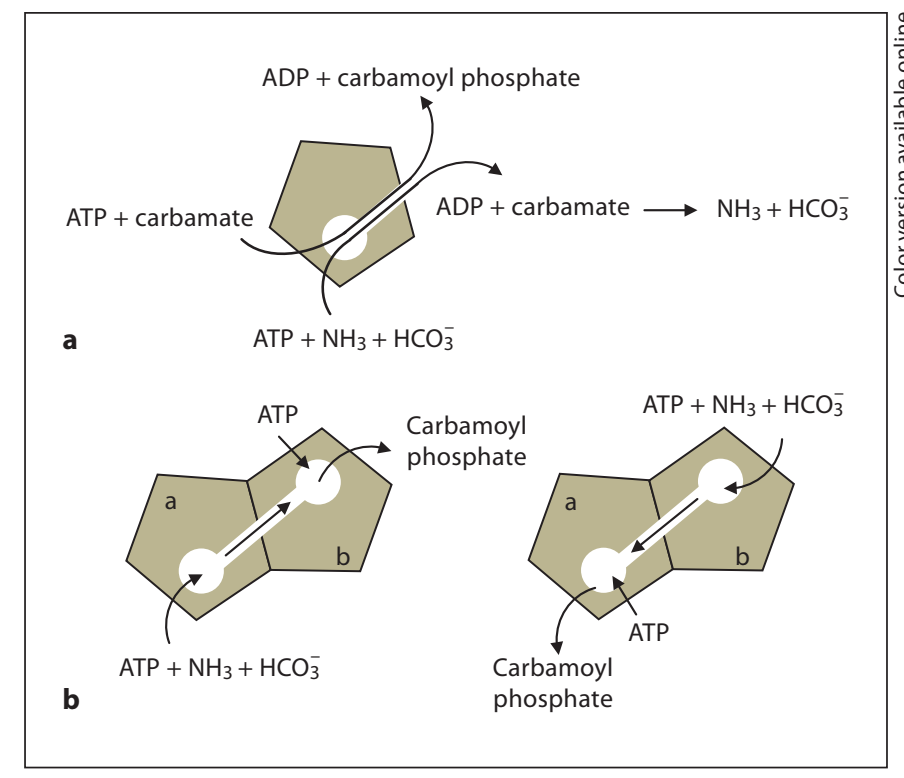

Fig. 6. Functional model of MS-s. Schematic representation of CP synthesis by MS-s. a Carbamate is synthesized from bicarbonate, ammonia, and ATP, released from the enzyme, and then binds once again where it is phosphorylated by a second ATP molecule forming CP. This process would be expected to be inefficient since carbamate once released from the complex would be degraded to bicarbonate and ammonia. $\mathbf{b}$ The synthesis of carbamate is catalyzed by one of the monomers in the MS-s dimer (a) which binds bicarbonate, ammonia, and ATP; the carbamate is sequestered and transferred to the second monomer (b) which catalyzes the phosphorylation of carbamate to form CP. In a subsequent catalytic cycle, the roles of subunits a and b may be reversed.

tulates that, as in E. coli CPSase, the two active sites are connected by an intramolecular tunnel. When ATP, ammonia, and bicarbonate bind to monomer ( $\mathrm{a}$ in fig. $6 \mathrm{~b}$ ) it becomes the site of synthesis of carbamate. Carbamate is then transferred to monomer $b$ which now becomes the $\mathrm{CP}$ synthesis domain by default. In a subsequent catalytic cycle the roles may be reversed such that subunit $b$ catalyzes carbamate synthesis and subunit a converts carbamate to CP. In this scheme, carbamate remains sequestered within the complex and protected from hydrolysis.

While further structural and biochemical studies are required to validate this model, there is precedence for this mechanism in mammalian CPSase. It was shown that both CPS.A and CPS.B domains of mammalian CAD separately cloned and expressed in E. coli could each dimerize and catalyze both ATP-dependent reactions and the overall synthesis of CP [Guy et al., 1997]. Moreover, the dissociation of CPS.A or CPS.B homodimers by high hydrostatic pressure abolished CP synthe- sis, while the dissociated subunits could still catalyze the CPSase partial reactions [Guy et al., 1998].

\section{Putative Physiological Function of MS-s}

In addition to MS-s, M. smithii possesses an active, full-length glutamine-dependent CPSase, MS-1 (unpubl. data), so the question arises of what selective advantage the unusual CPSase MS-s provides for the organism. Ammonia is the major source of nitrogen in M. smithii for the biosynthesis of nucleotides, amino acids, and many other metabolites. The organism has a specific ammonia transporter and two mechanisms for assimilation of ammonia, the glutamine synthetase-glutamate synthase and the glutamate dehydrogenase pathways [Samuel et al., 2007]. However, the availability of ammonia is strictly limited in the intestine and there is a competition for this scare resource between M. smithii and B. thetaiotaomicron. This conclusion is supported by several lines of evidence. For example, analytical studies [Samuel et al., 2007] demonstrated that, in M. smithii, the ratio of glutamine to 2-oxoglutarate, a good indication of the nitrogen status of the organism, was reduced 32-fold when M. smithii was cocolonized with $B$. thetaiotaomicron. Moreover, when both organisms are present in the intestine, there is an appreciable upregulation of the expression of enzymes involved in nitrogen assimilation in M. smithii [Samuel et al., 2007].

MS-s is a streamlined CPSase that is ideally suited to the task of capturing ammonia and converting it to pyrimidines and arginine. The kinetic studies showed that it has an appreciably higher affinity for ammonia than other CPSases. Moreover, the enzyme is also probably unregulated since the B3 or allosteric subdomain, the locus of regulation of all known CPSases, is not present in MS-s. While eukaryotic mitochondrial CPSase I, the enzyme that initiates the urea cycle, can be allosterically activated, there are no known allosteric inhibitors [Rubio and Cervera, 1995] presumably because it is essential in ureotylic animals to continuously and rapidly dispose of any ammonia formed via urea biosynthesis. Similarly, MS-s would be expected to be constitutively active if one of its major functions is to sequester ammonia, so that allosteric regulation would confer no advantage.

\section{Conclusions}

MS-s is a unique enzyme, the smallest functional CPSase discovered thus far and one that is only distantly related to other bacterial and archaeal CPSases. It consists of a catalytic domain but lacks the module involved in al- 
losteric regulation. The catalytically active species is an MS-s homodimer or larger species and the mechanism of $\mathrm{CP}$ synthesis appears to occur in three steps by the same mechanism utilized by the full-length prokaryotic and eukaryotic CPSases. CPSase are postulated to be derived from an ancestral kinase resembling MS-s. Thus, the characterization of this enzyme may provide a glimpse of the evolution of CPSases, which are thought to have arisen by gene duplication, fusion, and the recruitment of domains that confer regulatory functions. In addition to supplementing the function of the full-length CPSase MS-l by bolstering the supply of pyrimidine nucleotides and arginine, MS-s may provide an additional mechanism for harvesting ammonia, a scarce nutrient in the intestine. These minimal CPSases may be a characteristic of archaeal methanogens, since they are also present in M. thermoautotrophicus and M. stadtmanae. Considering that there is apparently no mammalian counterpart of this unusual protein, MS-s may be an attractive drug target in the treatment of human obesity.

\section{Experimental Procedures}

\section{Materials}

$P f u$ DNA polymerase, nucleotides, restriction enzymes, Bam$\mathrm{HI}$ and NcoI, thermosensitive alkaline phosphatase (FastAP), and T4 DNA ligase were from Fermentas Life Sciences; $\mathrm{Ni}^{2+}$-ProBond resin was purchased from Invitrogen; aspartate, carbamoyl aspartate, antipyrine, diacetyl monoxime, triethoanolamine (TEA), phosphoenol pyruvate, dimethyl suberimidate, and the enzymes pyruvate kinase, lactate dehydrogenase, hexokinase, and glucose 6-phosphate dehydrogenase were from Sigma-Aldrich; A. aeolicus aspartate transcarbamoylase (ATCase) recombinant enzyme was expressed in E. coli and purified as previously described [Purcarea et al., 2003].

\section{Strains and Plasmids}

The M. smithii ATCC35061 strain (DSM 861) was from the DSMZ Bacteria Collection [Samuel et al., 2007]. The E. coli DH5 $\alpha$ and BL21(DE3) strains and the pRSETC expression vector were from Invitrogen.

\section{M. smithii Growth}

The methanogen M. smithii ATCC35061 was cultivated anaerobically at $37^{\circ} \mathrm{C}$ in DSMZ 119 medium containing fatty acids and supplemented with DSMZ 320 salt solutions, in the presence of an $80 \% \mathrm{H}_{2}: 20 \% \mathrm{CO}_{2}$ gas mixture [Samuel et al., 2007]. Cultures of $20 \mathrm{ml}$ were inoculated with 1-ml cultures under an $\mathrm{N}_{2}$ atmosphere. The flasks were flushed every $24 \mathrm{~h}$ with fresh $\mathrm{H}_{2}: \mathrm{CO}_{2}$ mixture, and cell viability was analyzed by microscopy.

\section{Cloning and Expression}

M. smithii chromosomal DNA was isolated from a $20-\mathrm{ml}$ culture using a DNeasy Blood and Tissue Kit for genomic DNA extraction (Qiagen), with an additional cell lysis step using 50 units of mutanolysin (Sigma). The carB1 gene (YP_001272934) from $M$. smithii was amplified by PCR using 2.5 units of Pfu DNA polymerase, $170 \mathrm{ng}$ of $M$. smithii chromosomal DNA as a template, and 100 pmol of 5MS-s (5'-GGAGATTGGATCCAAATTTTATTTAT-CGGTTCAAG-3') and 3MS-s (5'-GTGTTTAAGCCATGG TTA-AATCAAATCTTCAACATATC-3') primers defining the $5^{\prime}$ and $3^{\prime}$ ends of the $M$. smithii carB1 gene, respectively. The DNA fragment was amplified after 30 cycles of $95^{\circ} \mathrm{C}$ for $45 \mathrm{~s}, 52^{\circ} \mathrm{C}$ for $1 \mathrm{~min}$, and $72^{\circ} \mathrm{C}$ for 3 min each cycle, and purified with a DNA Clean \& Concentrator Kit (Zymo Research). After digestion with $\mathrm{BamHI} / \mathrm{NcoI}$, the gene (750 ng) was inserted into the pRSETC expression vector (130 $\mathrm{ng}$ ) digested with the same restriction enzymes, and dephosphorylated with thermosensitive alkaline phosphatase. Both strands of the resulting construct were sequenced using a Genetic Analyzer 3500 (Applied Biosystems). The M. smithii CPS gene was expressed in E. coli BL21(DE3) after a 5 -hour induction at $20^{\circ} \mathrm{C}$ with $1 \mathrm{mM}$ IPTG.

\section{Purification}

The recombinant $M$. smithii enzyme expressed in E. coli as an $\mathrm{N}$-terminal fused protein with a $3-\mathrm{kDa}$ His-tag polypeptide was purified in one step at $4^{\circ} \mathrm{C}$ by affinity chromatography. Cells from a 100 -ml culture were resuspended in $3 \mathrm{ml}$ of $50 \mathrm{mM}$ Tris $\mathrm{HCl}, \mathrm{pH}$ 8 , containing $2 \mathrm{mM} 2$-mercaptoethanol and 1:100 protease inhibitor cocktail (Sigma), and disrupted by sonication 6 times for $30 \mathrm{~s}$ using a Sonifier Cell disruptor W-350 (Bronson Sonic Power). The cell extract was centrifuged at 20,000 $\mathrm{g}$ for $30 \mathrm{~min}$, and the supernatant was applied to a $1-\mathrm{ml} \mathrm{Ni}^{2+}$-ProBond column equilibrated with $50 \mathrm{~mm}$ TrisHCl, $\mathrm{pH}$ 8, and $200 \mathrm{mM} \mathrm{NaCl}$ (TN buffer). After 20 - $\mathrm{ml}$ washes with the same buffer, the enzyme was eluted with $1-\mathrm{ml}$ fractions of TN buffer containing increasing $(50,100,200$, and $500 \mathrm{~mm}$ ) imidazole concentrations. The elution fractions were analyzed by electrophoresis on $12 \%$ SDS-PAGE gels. Fractions containing pure recombinant CPSase were collected and the buffer was changed to $50 \mathrm{mM}$ Tris $\mathrm{HCl}, \mathrm{pH}$ 8, and $5 \mathrm{mM} 2$-mercaptoethanol, using PD-10 desalting columns (GE Healthcare), to eliminate imidazole and $\mathrm{NaCl}$.

Chemical Cross-Linking

Fractions containing $20 \mu \mathrm{g}$ M. smithii CPSase in $100 \mathrm{~mm}$ triethanolamine, $\mathrm{pH} 8.5$, and $100 \mathrm{mM} \mathrm{NaCl}$ were incubated with 10 mM dimethyl suberimidate [Davies et al., 1970] for 0, 5, 10, and 15 $\mathrm{min}$ at room temperature in a total volume of $45 \mu \mathrm{l}$. The reaction was stopped by addition of $5 \mu \mathrm{l}$ of $1 \mathrm{M}$ TrisHCl, $\mathrm{pH} 8$, and the cross-linked proteins were visualized by SDS-PAGE (10\% gels).

\section{Size Exclusion Chromatography}

Size exclusion chromatography was carried out at $4^{\circ} \mathrm{C}$ using a Hiload 16/60 Superdex 200 prep grade column equilibrated with $50 \mathrm{~mm}$ TrisHCl, pH 8, containing $5 \mathrm{~mm} 2$-mercaptoethanol. The column was loaded with $500-\mu \mathrm{l}(5 \mathrm{mg})$ of the purified MS-s, and the protein was eluted at $1 \mathrm{ml} / \mathrm{min}$ with the equilibration buffer, monitoring the absorbance at $280 \mathrm{~nm}$. The fractions containing the protein peaks were analyzed by SDS-PAGE.

\section{CPSase Assay}

Ammonia-dependent CPSase activity was measured using the radioactive assay in which the CPSase reaction is coupled with $A$. aeolicus aspartate transcarbamoylase (ATCase) [Ahuja et al., 2001]. Expression and purification of the A. aeolicus ATCase were 
carried out as previously described [Purcarea et al., 2003]. The reaction mixture contained $100 \mathrm{mM}$ sodium $\left[{ }^{14} \mathrm{C}\right]$ bicarbonate (100 dpm/ $\mu \mathrm{mol}), 20 \mathrm{mM}$ ATP, $22 \mathrm{mM} \mathrm{MgCl}_{2}$ and $200 \mathrm{mM}$ ammonium chloride, $50 \mathrm{mM}$ Tris $\mathrm{HCl}, \mathrm{pH} 8.0,100 \mathrm{mM} \mathrm{KCl}$, in the presence of $6 \mathrm{~mm}$ aspartate, $\mathrm{pH} \mathrm{7}$, and $1 \mu \mathrm{g}$ of purified A. aeolicus ATCase and various concentrations of recombinant MS-s CPSase, in a total volume of $0.3 \mathrm{ml}$. After a 20 -min incubation at $37^{\circ} \mathrm{C}$, the reaction was stopped by addition of $0.7 \mathrm{ml} 10 \%$ trichloracetic acid. After evaporation of the liquid phase at $95^{\circ} \mathrm{C}$ to decompose the unreacted bicarbonate, the radioactivity incorporated in the stable reaction product carbamoyl aspartate was measured after addition of $8 \mathrm{ml}$ Aquasol scintillation liquid. Alternatively, the carbamoyl aspartate formed in the coupled reaction was measured by a colorimetric assay previously described [Purcarea et al., 2001].

\section{Partial Reactions}

The bicarbonate-dependent ATPase reaction was measured using a pyruvate kinase/lactate dehydrogenase coupled assay [Post et al., 1990] by monitoring the rate of ADP synthesis in the presence and absence of ammonium chloride. The reaction was performed in a final volume of $0.5 \mathrm{ml}$ containing $100 \mathrm{mM}$ sodium bicarbonate, $20 \mathrm{~mm}$ ATP, $22 \mathrm{mM} \mathrm{MgCl}$, $50 \mathrm{~mm}$ TrisHCl, pH 8.0, $100 \mathrm{~mm} \mathrm{KCl}, 1 \mathrm{~mm}$ phosphoenolpyruvate, $0.2 \mathrm{mM} \mathrm{NADH}, 10$ units of pyruvate kinase, and 20 units of lactate dehydrogenase, and, optionally, $200 \mathrm{~mm}$ ammonium chloride. The reaction rate was measured spectrophotometrically at $340 \mathrm{~nm}$, and the concentration was calculated using $\varepsilon_{\mathrm{NADH}}=6.22 \mathrm{M}^{-1} \cdot \mathrm{cm}^{-1}$. The CP-dependent ATP synthetase activity was assayed by measuring the rate of ATP formation from ADP and $\mathrm{CP}$ in the coupled reaction with hexokinase/glucose-6-phosphate dehydrogenase, following a similar procedure [Miran et al., 1991]. The reaction mixture contained $50 \mathrm{~mm}$ TrisHCl, $\mathrm{pH} 8.0,100 \mathrm{~mm} \mathrm{KCl}, 22 \mathrm{~mm} \mathrm{MgCl}_{2}$, $1 \mathrm{mM}$ NAD, $20 \mathrm{mM}$ ADP, $2 \mathrm{mM} \mathrm{CP}, 5 \mathrm{mM}$ glucose, 20 units of hexokinase, and 10 units of glucose-6-phosphate dehydrogenase in a final volume of $0.5 \mathrm{ml}$. Both partial reactions were assayed at $22^{\circ} \mathrm{C}$ and the initial rate was calculated from the time course up to a 5 -min time course.
Structure Analysis and Molecular Modeling

The genes encoding the CPSase subunits in the M. smithii ATCC35061 genome sequence were identified by BLAST-NCBI genome database screening. Sequence analysis was performed using the genomic and proteomic software package ExPASy SIB Bioinformatics Resource Portal. Protein pair alignment was carried out with EMBOSS needle alignment (EMBL-EBI) and multiple alignment was performed using ClustalW. Amino acid sequence randomization was performed using random protein sequence generator RandSeq software (ExPaSy). Modeling of the three-dimensional structure was carried out using Modeller 9v9 [Sali and Blundell, 1993], based on the 1BXR X-ray structure of $E$. coli CPSase in complex with the ATP analog AMPPNP [Thoden et al., 1999]. Fifty models were generated and the best candidate was chosen based on DOPE (discrete optimized protein energy), zDOPE (normalized discrete optimized protein energy), and GA341 scores. The energy minimization was performed with Gromos 96 implemented in Swiss PDB Viewer. The model was further refined by loop optimization using ModLoop [Fiser and Sali, 2003] followed by energy minimization. In every round of optimization, at least 10 models where generated, followed by energy minimization. The quality of the model was assessed by the Ramachandran plot obtained with PROCHEK [Laskowski et al., 1993], ERRAT2 [Colovos and Yeates, 1993], and ProSa [Sippl, 1993] programs. Superposition of the MS-s model with 1BXR and calculation of the root mean square deviation were obtained using Swiss PDB Viewer [Guex and Peitsch, 1997]. The structures where visualized using the PyMol Molecular Graphics System 0.99rc6 (Schrodinger, LLC).

\section{Acknowledgments}

We thank Asmita Vaishnav for technical expertise and assistance. This work was supported by ANCS-UEFISCDI Romania, PNII ID_1034 (contract No. 1023/2009), NIH R01 grant GM/ CA60371, and the Wayne State University Provost's Research Stimulation Award.

\section{References}

- Ahuja A, Purcarea C, Guy HI, Evans DR: A novel carbamoyl phosphate synthetase from Aquifex aeolicus. J Biol Chem 2001;276: 45694-45703.

Alonso E, Rubio V: Affinity cleavage of carbamoyl-phosphate synthetase I localizes regions of the enzyme interacting with the molecule of ATP that phosphorylates carbamate. Eur J Biochem 1995;229:377-384.

-Anderson PM: Carbamoyl-phosphate synthetase: an example of effects on enzyme properties of shifting an equilibrium between active monomer and active oligomer. Biochemistry 1986;25:5576-5582.

-Anderson PM, Meister A: Evidence for an activated form of carbon dioxide in the reaction catalyzed by Escherichia coli carbamyl phosphate synthetase. Biochemistry 1965;4: 2803-2809.
Balch WE, Fox GE, Magrum LJ, Woese CL, Wolf RS: Methanogens: reevaluation of a unique biological group. Microbiol Rev 1979;43: 260-296.

Belay N, Johnson R, Rajagopal BS, De Macario ES, Daniels L: Methanogenic bacteria from human dental plaque. Appl Environ Microbiol 1988;54:600-603.

-Belay N, Mukhopadhyay B, De Macario EC, Galask R, Daniels L: Methanogenic bacteria in human vaginal samples. J Clin Microbiol 1990;28:1666-1668.

Buck S, Hansen EE: Genomic and metabolic adaptations of Methanobrevibacter smithii to the human gut. Proc Natl Acad Sci USA 2007;104:10643-10648.

Bult CJ, White O, Olsen GJ, Zhou L, Fleischmann RD, Sutton GG, Blake JA, FitzGerald LM, Clayton RA, Gocayne JD, Kerlavage AR,
Dougherty BA, Tomb FJ, Adams MD, Reich CI, Overbeek R, Kirkness EF, Weinstock KG, Merrick JM, Glodek A, Scott JL, Geoghangen NS, Venter JC: Complete genome sequence of the methanogenic archaeon, Methanococcus jannaschii. Science 1996; 273:1058-1073.

Colovos C, Yeates TO: Verification of protein structures: patterns of nonbonded atomic interactions. Protein Sci 1993;2:1511-1519.

Davies GE, Stark GR: Dimethyl suberimidate, a cross-linking reagent, in studying the subunit structure of oligomeric proteins. Proc Natl Acad Sci USA 1970;66:651-656.

Dridi B, Raoult D, Drancourt M: Archaea as emerging organisms in complex human microbiomes. Anaerobe 2011;17:56-63.

-Durbecq V, Legrain C, Roovers M, Piérard A, Glansdorff N: The carbamate kinase-like 
carbamoyl phosphate synthetase of the hyperthermophilic archaeon Pyrococcus furiosus, a missing link in the evolution of carbamoyl phosphate biosynthesis. Proc Natl Acad Sci USA. 1997;94:12803-12808.

- Fiser A, Sali A: ModLoop: automated modeling of loops in protein structures. Bioinformatics 2003;19:2500-2501.

-Fresquet V, Mora P, Rochera L, Ramón-Maiques $S$, Rubio V, Cervera J: Site-directed mutagenesis of the regulatory domain of Escherichia coli carbamoyl phosphate synthetase identifies crucial residues for allosteric regulation and for transduction of the regulatory signals. J Mol Biol 2000:299:979-991.

Guex N, Peitsch MC: SWISS-MODEL and the Swiss-PdbViewer: an environment for comparative protein modeling. Electrophoresis 1997; 18:2714-2723.

-Guillou F, Rubino SD, Markovitz RS, Kinney DM, Lusty CJ: Escherichia coli carbamoylphosphate synthetase: domains of glutaminase and synthetase subunit interaction. Proc Natl Acad Sci USA 1989;86:8304-8308.

-Guy HI, Bouvier A, Evans DR: The smallest carbamoyl-phosphate synthetase. J Biol Chem 1997;272:29255-29262.

Guy HI, Evans DR: Function of the major synthetase subdomains of carbamyl-phosphate synthetase. J Biol Chem 1996;271:1376213769 .

-Guy HI, Schmitt B, Hervé G, Evans DR: Pressure-induced dissociation of carbamoylphosphate synthetase domains: the catalytically active form is dimeric. J Biol Chem 1998;273:14172-14178.

Horz HP, Conrads G: The discussion goes on: what is the role of Euryarchaeota in humans. Archaea DOI: 10.1155/2010/967271.

-Huang X, Raushel FM: An engineered blockage within the ammonia tunnel of carbamoyl phosphate synthetase prevents the use of glutamine as a substrate but not ammonia. Biochemistry 2000;39:3240-3247.

-Kim J, Raushel FM: Allosteric control of the oligomerization of carbamoyl phosphate synthetase from Escherichia coli. Biochemistry 2001;40:11030-11036.

Kothe M, Purcarea C, Guy HI, Evans DR, Powers-Lee SG: A Novel carbamoyl-phosphate synthetase from Aquifex aeolicus. J Biol Chem 2005;14:37-44.

Laskowski RA, MacArthur MW, Moss DS, Thornton JM: PROCHECK: a program to check the stereochemical quality of protein structures. J Appl Crystallogr 1993;26:283291.

- Legrain C, Demarez M, Glansdorff N, Pierard A: Ammonia-dependent synthesis and metabolic channelling of carbamoyl phosphate in the hyperthermophilic archaeon Pyrococcus furiosus. Microbiology 1995;141:1093-1099.
Marina A, Uriarte M, Barcelona B, Fresquet V, Cervera J, Rubio V: Carbamate kinase from Enterococcus faecalis and Enterococcus faecium: cloning of the genes, studies on the enzyme expressed in Escherichia coli, and sequence similarity with $N$-acetyl-L-glutamate kinase. Eur J Biochem 1998;253:280-291.

Meister A: Mechanism and regulation of the glutamine-dependent carbayl phosphate synthetase of Escherichia coli. Adv Enzymol Relat Areas Mol Biol 1989;62:315-374.

Miller TL, Wolin MJ, De Macario EC, Macario AJ: Isolation of Methanobrevibacter smithi from human feces. Appl Environ Microbiol 1982;43:227-232.

Million M, Maraninchi M, Henry M., Armougom F, Richet $\mathrm{H}$, Carrieri P, Valero R, Raccah D, Vialettes B, Raoult D: Obesity-associated gut microbiota is enriched in Lactobacillus reuteri and depleted in Bifidobacterium animalis and Methanobrevibacter smithii. Int J Obes DOI: 10.1038/ijo.2011.153.

Miran SG, Chang SH, Raushel FM: Role of the four conserved histidine residues in the amidotransferase domain of carbamoyl phosphate synthetase. Biochemistry 1991;30 7901-7909.

Nava GM, Caronero F, Croix JA, Greenberg E, Gaskins HR: Abundance and diversity of mucosa-associated hydrogenotrophic $\mathrm{mi}$ crobes in the healthy human colon. ISME DOI: 10.1038/ismej.2011.90.

Nyunoya H, Lusty CJ: The carB gene of Escherichia coli: a duplicated gene coding for the large subunit of carbamoyl-phosphate synthetase. Proc Natl Acad Sci USA 1983;80 4629-4633.

Paulus TJ, Switzer RL: Caracterization of pyrimidine-repressible and arginine-repressible carbamyl phosphate synthetase from Bacillus subtilis. J Bacteriol 1979;137:82-91.

Plottel CS, Blaser MJ: Microbiome and malig nancy. Cell Host Microb 2011;10:324-335.

Popa E, Rusu A, Zamfir M, Dumitru L, Purcarea C: An ammonia-metabolizing enzyme from the human archaeon Methanobrevibacter smithii might represent a missing link in the evolution of carbamoyl phosphate synthetases. Biotechnol Biotechnol Equip 2009;23. 533-537.

Post LE, Post DJ, Raushel FM: Dissection of the functional domains of Escherichia coli carbamoyl phosphate synthetase by site-directed mutagenesis. J Biol Chem 1990;265:77427747

- Purcarea C, Ahuja A, Lu T, Kovari L, Guy HI, Evans DR: Aquifex aeolicus aspartate transcarbamoylase, an enzyme specialized for the efficient utilization of unstable carbamoyl phosphate at elevated temperature. J Biol Chem 2003;278:52924-52934.

- Purcarea C, Hervé G, Cunin R, Evans DR: Cloning, expression and structure analysis of carbamat kinase-like carbamoyl phosphate synthetase from Pyrococcus abyssi. Extremophiles 2001;5:229-239.
Purcarea C, Simon V, Prieur D, Hervé G: Purification and characterization of carbamylphosphate synthetase from the deep-sea hyperthermophilic archaebacterium Pyrococcus abyssi. Eur J Biochem 1996;236:189-199.

Rubio V, Cervera J: The carbamoyl-phosphat synthetase family and carbamate kinase: structure-function studies. Biochem Soc Trans 1995;23:879-883.

Rubio V, Cervera J, Lusty CJ, Bendala E, Britton HG. Domain structure of the large subunit of Escherichia coli carbamoyl phosphate synthetase: location of the binding site for the allosteric inhibitor UMP in the COOH-terminal domain. Biochemistry 1991 30:10681075.

Rubio SD, Nyunoya H, Lusty CJ: In vivo synthesis of carbamyl phosphate from $\mathrm{NH} 3$ by the large subunit of Escherichia coli carbamyl phosphate synthetase. J Biol Chem 1987;262: 4382-4386

-Šali A, Blundell TL: Comparative protein modelling by satisfaction of spatial restraints. J Mol Biol 1993;234:779-815

Samuel BS, Gordon JI: A humanized gnotobiotic mouse model of host-archaeal-bacterial mutualism. Proc Natl Acad Sci USA 2006;103: 10011-10016.

Samuel BS, Hansen EE, Manchester JK, Coutinho PM, Henrissat B, Fulton R, Latreille P, Kim K, Wilson RK, Gordon JI: Genomic and metabolic adaptations of Methanobrevibacter smithii to the human gut. Proc Natl Acad Sci USA 2007;104:10643-10648.

Sippl MJ: Recognition of errors in three-dimensional structures of proteins. Proteins 1993; 17:355-362

Stams AJ: Metabolic interactions between anaerobic bacteria in methanogenic environments. Antonie van Leeuwenhoek 1994;66: 271-294.

Thoden JB, Holden HM, Wesenberg G, Raushel FM, Rayment I: The structure of carbamoyl phosphate synthetase: a journey of $96 \AA$ from substrate to product. Biochemistry 1997;36: 6305-6316.

Thoden JB, Wesenberg G, Raushel FM, Holden HM: Carbamoyl phosphate synthetase: closure of the B-domain as a result of nucleotide binding. Biochemistry 1999;38:2347-2357.

Trotta PP, Burt ME, Haschemeyer RH, Meister A: Reversible dissociation of carbamyl phosphate synthetase into a regulated synthesis subunit and a subunit required for glutamine utilization. Proc Natl Acad Sci USA 1971;68: 2599-2603.

Uriarte M, Marina A, Ramon-Maiques S, Rubio V, Durbecq V, Legrain C, N. Glansdorff N: Carbamoyl phosphate synthesis: carbamate kinase from Pyrococcus furiosus. Methods Enzymol 2001;331:236-247.

- Yang H, Park SM, Nolan WG, Lu CD, Abdelal AT: Cloning and characterization of the arginine-specific carbamoyl-phosphate synthetase from Bacillus stearothermophilus. Eur J Biochem 1997;249:443-449. 